\title{
Eficiência da absorção, translocação e uso de N e P pela Pfaffia glomerata (Spreng.) Pedersen
}

\author{
SERRA, A.P. ${ }^{1 *}$; MARCHETTI, M.E. ${ }^{2}$; VIEIRA, M.C. ${ }^{2}$; ROBAINA, A.D. ${ }^{2}$; NASCIMENTO, J.M. ${ }^{2}$; VERONESI, C. ${ }^{2}$; \\ MATOS, $\mathrm{F}^{2}$ \\ ${ }^{1}$ Empresa Brasileira de Pesquisa Agropecuária, Centro Nacional de Pesquisa de Gado de Corte, EMBRAPA-CNPGC, \\ Vila Popular, Caixa Postal 154, CEP: 79002-970, Campo Grande-Brasil *ademar.serra@cnpgc.embrapa.br. \\ 2Universidade Federal da Grande Dourados, Pós Graduação em Agronomia - Produção Vegetal, FCA/UFGD - \\ Caixa Postal 533, CEP: 79804-970, Dourados-Brasil
}

RESUMO: Tendo como objetivo avaliar a eficiência de absorção, translocação e uso de nitrogênio $(\mathrm{N})$ e fósforo $(\mathrm{P})$ pela Pfaffia glomerata, foi conduzido o presente trabalho em casa de vegetação no delineamento inteiramente casualizado com três repetições. Os tratamentos foram dispostos em esquema fatorial $5 \times 5$, sendo cinco doses de $\mathrm{N}\left(0,125,250,375\right.$ e $500 \mathrm{mg}$ vaso- $\left.{ }^{-1}\right)$ e cinco doses de $\mathrm{P}\left(\mathrm{P}_{2} \mathrm{O}_{5}\right)\left(0,125,250,375\right.$ e $\left.500 \mathrm{mg} \mathrm{vaso}^{-1}\right)$. Os resultados evidenciaram que a eficiência de absorção de $\mathrm{Ne} P$ foi influenciada pelas doses de $\mathrm{Ne} P$ utilizadas no experimento apresentando interação entre os fatores em estudo. A eficiência de translocação do $\mathrm{N}$ foi consideravelmente menor que a de $\mathrm{P}$, provavelmente pela maior concentração de compostos nitrogenados nas raízes em detrimento da parte aérea. Observou-se maior eficiência de uso do $\mathrm{P}$ devido à menor concentração desse elemento na planta. A absorção de $\mathrm{N}$ e de $\mathrm{P}$ foi influenciada pela dose desses elementos no solo, sendo possível a estimativa das doses desses nutrientes que proporcionem maiores eficiências de absorção, translocação, uso e teor total.

Palavras-chave: eficiência nutricional, nutrição de plantas, ginseng brasileiro, nitrogênio

ABSTRACT: Efficiency of $\mathrm{N}$ and $\mathrm{P}$ uptake, translocation and use by Pfaffia glomerata (Spreng.) Pedersen. Aimed at evaluating the efficiency of nitrogen $(N)$ and phosphorus $(P)$ uptake, translocation and use by Pfaffia glomerata, the present study was conducted in greenhouse, in completely randomized design with three replicates. Treatments were displayed in $5 \times 5$ factorial design, with five $\mathrm{N}$ levels $\left(0,125,250,375\right.$ and $\left.500 \mathrm{mg} \mathrm{pot}^{-1}\right)$ and five $\mathrm{P}$ $\left(\mathrm{P}_{2} \mathrm{O}_{5}\right)$ levels $\left(0,125,250,375\right.$ and $\left.500 \mathrm{mg} \mathrm{pot}^{-1}\right)$. Results evidenced that $\mathrm{N}$ and $\mathrm{P}$ uptake efficiency was influenced by the $\mathrm{N}$ and $\mathrm{P}$ levels used in the experiment, showing interaction among the studied factors. $\mathrm{N}$ translocation efficiency was considerably lower than that of $\mathrm{P}$, probably due to the lower concentration of nitrogenous compounds in the roots than in the shoot. P use efficiency was higher due to the lower concentration of this element in the plant. $\mathrm{N}$ and $\mathrm{P}$ uptake was influenced by the level of these elements in the soil, being possible to estimate the levels of these nutrients which provide higher uptake, translocation and use efficiency besides total content.

Key words: nutritional efficiency, plant nutrition, Brazilian ginseng, nitrogen

\section{INTRODUÇÃO}

O cultivo de plantas medicinais tornou-se alternativa interessante e rentável na agricultura brasileira (Simões et al., 2000) justificando a necessidade de se conhecer as exigências nutricionais da planta para cultivos comerciais. $O$ aumento na produtividade em plantas medicinais, assim como a biossíntese de compostos de valor econômico estão correlacionados com a otimização da nutrição mineral (Mairapetyan et al., 1999).

Considerando a intensa exploração predatória das reservas naturais destas espécies, justifica-se a elaboração de planos de manejo ou projetos de cultivo (Montanari et al., 1999).

O produto comercial de interesse da planta

Recebido para publicação em 07/07/2009

Aceito para publicação em 25/01/2012

Rev. Bras. PI. Med., Botucatu, v.14, n.2, p.255-260, 2012. 
de Pfaffia se encontra nas raízes, sendo extraído compostos como ecdisterona (Fenner et al., 2008; Flores et al., 2009a) e $\beta$-ecdisona (Corrêa Junior et al., 2008; Flores et al., 2009b). Os compostos medicinais das raízes de Pfaffia podem ser utilizados como tônico afrodisíaco e antidiabético (Montanari Junior et al., 1999; Magalhães, 2000).

A $P$. glomerata é planta perene, ereta ou semi-ereta, com 0,5 a 2,5 $\mathrm{m}$ de altura, possui raiz tuberosa e, geralmente, bifurcada, como do "ginseng" do Oriente, daí o nome "ginseng brasileiro" ou "ginseng-do-pantanal". As folhas são opostas, quando novas são largas e aquelas próximas das inflorescências são estreitas. A planta floresce e frutifica de setembro a maio e, às vezes, nos outros meses na região do Pantanal Sul Matogrossense (Pott \& Pott, 1994).

$O$ nitrogênio tem ação controversa em plantas medicinais, pois, a deficiência proporciona em papoula (Papaver somniferum) e em beladona (Atropa beladona) o aumento na concentração de alcalóides, enquanto que na lobélia (Lobelia inflata) ocorre redução. Em camomila (Chamomila recutila) o nitrogênio em interação com o potássio proporciona aumento no rendimento em óleo essencial por unidade de área. Martins et al. (1998) citam que o nitrogênio deve ser fornecido principalmente por meio da adubação orgânica, sem excessos, pois pode trazer problemas na produção de substâncias ativas, como ocorre na losna (Artemisia absinthium).

O fósforo $(P)$ se encontra na planta na forma de $\mathrm{H}_{2} \mathrm{PO}_{4}$, podendo permanecer como fosfato inorgânico livre ou esterificado dentro de grupo hidroxila de cadeia de carbono (C-O-P) como um simples éster fosfato ou preso a outro fosfato adenosinadifosfática (ADP) e formando a adenosinatrifosfática (ATP) (Marschner, 1995; Malavolta, 2006; Epstein \& Bloom, 2006).

Níveis adequados de $P$ nas plantas medicinais podem favorecer a produção de alcalóides e outros princípios ativos, sendo que a deficiência de $\mathrm{P}$ na planta pode causar redução na produção de biomassa e tendo como resultado negativo a redução na produção de metabólitos (Martins et al., 1998).

Portanto, a avaliação da eficiência de uso, absorção e translocação de $\mathrm{N}$ e $\mathrm{P}$ nas plantas medicinais podem constituir importante ferramenta para o esclarecimento de alguns aspectos nutricionais contribuindo para a adequação do manejo da adubação. Assim, o objetivo em realizar esse trabalho foi avaliar a eficiência de absorção, translocação e uso de $\mathrm{N}$ e $\mathrm{P}$ pela $P$. glomerata, em função da adubação nitrogenada e fosfatada.

\section{MATERIAL E MÉTODO}

O experimento foi desenvolvido utilizando a espécie Pfaffia glomerata (Spreng.) Pedersen, cultivada em casa de vegetação na Faculdade de Ciências Agrárias - FCA, da Universidade Federal da Grande Dourados - UFGD, em Dourados (22ำ 12' de latitude Sul e 54은 $56^{\prime}$ de longitude Oeste, altitude de 452 m), estado de Mato Grosso do Sul - MS, Brasil.

O solo utilizado como substrato foi Latossolo Vermelho distroférrico (Embrapa, 2006), coletado em área de vegetação natural de cerrado, na camada de $0-20 \mathrm{~cm}$. O solo amostrado foi seco ao ar, destorroado e passado em peneira com malha de $2 \mathrm{~mm}$. Três subamostras foram retiradas desse material para a determinação das características químicas, apresentadas na Tabela 1.

Alíquotas de $6 \mathrm{Kg}$ desta terra foram empregadas como substrato, dispostas em vasos, de modo que a unidade experimental foi constituída por um vaso contendo uma planta de $P$. glomerata.

Os fatores em estudo foram cinco doses de $\mathrm{N}$ (mg vaso-1): $0,125,250,375$ e 500 , fornecidas na forma de uréia, e cinco doses de $\mathrm{P}_{2} \mathrm{O}_{5}\left(\mathrm{mg} \mathrm{vaso}^{-1}\right)$ : 0 , $125,250,375$ e 500 , sendo utilizada como fonte de $\mathrm{P}_{2} \mathrm{O}_{5}$ o superfosfato triplo. $\mathrm{O}$ delineamento experimental foi o inteiramente casualizado com os tratamentos dispostos em esquema fatorial $5 \times 5$, com três repetições.

Com base nos resultados da análise do solo realizou-se a correção da acidez, por meio da adição de calcário dolomítico finamente moído (PRNT = $100 \%$ ), na dose de $16 \mathrm{~g}$ vaso ${ }^{-1}$, sendo esta quantidade necessária para elevar a saturação por bases a $70 \%$.

Os vasos foram mantidos úmidos com água destilada visando manter a umidade equivalente a $60 \%$ do volume total de poros. O volume de água aplicado foi estimado com base na densidade aparente (Dap) e no volume de poros (Vp) (EMBRAPA, 1997),

TABELA1. Característica química do solo (Latossolo Vermelho distroférrico) utilizado no experimento.

\begin{tabular}{|c|c|c|c|c|c|c|c|c|c|c|}
\hline pH & M.O. & $\mathbf{P}$ & K & $\mathrm{Ca}$ & Mg & Al & $\mathrm{H}+\mathrm{Al}$ & SB & $T$ & V \\
\hline $\mathrm{CaCl}_{2}$ & $\mathrm{~g} \mathrm{dm}^{-3}$ & $\mathrm{mg} \mathrm{dm}^{-3}$ & & - & $-\mathrm{cn}$ & $\mathrm{ol}_{\mathrm{c}} \mathrm{dm}$ & -7 & - & & -\%- \\
\hline 3,9 & 16,9 & 1,0 & 0,03 & 0,6 & 0,1 & 1,82 & 9,4 & 0,73 & 10,13 & 7 \\
\hline
\end{tabular}

Laboratório de Fertilidade do Solo da FCA/UF1,0 GD, segundo as especificações da Embrapa (1997).

Rev. Bras. Pl. Med., Botucatu, v.14, n.2, p.255-260, 2012. 
permanecendo os vasos incubados por 45 dias.

A semeadura da $P$. glomerata foi realizada em bandejas sem células, no Horto de Plantas Medicinais da FCA/UFGD. O substrato utilizado para a germinação foi uma mistura de solo, areia e casca de arroz carbonizada.

As mudas de $P$. glomerata foram transplantadas para os vasos 40 dias após a germinação, ocasião em que foram aplicados os tratamentos com as doses de $\mathrm{N}$ e $\mathrm{P}$, nas doses indicadas anteriormente, sendo o $\mathrm{N}$ aplicado em solução, 1/3 no momento do transplante e 2/3 restantes aplicados 15 dias após.

Oito meses após o transplantio as plantas foram cortadas rente ao solo e separadas em parte aérea e radicular. Em seguida, as amostras da parte aérea e radicular das plantas foram lavadas com água deionizada, secas em estufa com circulação forçada de ar a 65ㄷ $\mathrm{C}$ até massa constante para determinação da massa seca da parte aérea e radicular. A biomassa seca foi moída em moinho tipo Willey e acondicionada em sacos plásticos para a realização das análises químicas.

A determinação do $\mathrm{N}$ foi realizada por digestão sulfúrica pelo método microkjeldhal e nitricoperclórico, enquanto a análise de $\mathrm{P}$ seguiu a metodologia descrita por Malavolta et al. (1997).

A partir da massa seca e do conteúdo de $\mathrm{N}$ e $P$ na planta foram calculadas as eficiências de uso (EFU), de absorção (EFA) e de translocação (EFT) para $\mathrm{N} \mathrm{e} \mathrm{P}$, como pode ser observado nas fórmulas (a), (b), (c) e (d):

(a)EFU $\left(g^{2} \mathrm{mg}\right)=\frac{(\text { massa seca total produzida })^{2}}{\text { conteúdo total do nutriente na planta }}$ (Siddiqi \& Glass, 1981);

(b)EFA $\left(\mathrm{mg} \mathrm{g}^{1}\right)=\frac{\text { conteúdo total do nutriente na planta }}{\text { massa seca de raízes }}$ (Swiader et al., 1994);

(c)EFT $(\%)=\left[\frac{\text { conteúdo do nutriente na parte aérea }(\mathrm{mg})}{\text { conteúdo do nutriente na parte aérea }(\mathrm{mg})}\right] \cdot 100$ (Li et al., 1991);

(d) $\begin{aligned} & \text { conteúdo total do } \\ & \text { nutriente }\left(\mathrm{mg} \mathrm{planta}^{-1}\right)\end{aligned}=\sum \begin{aligned} & \text { nutriente na parte } \\ & \text { aérea e radicular }\end{aligned}$

Os resultados da eficiência nutricional (EFU, EFA e EFT) do $\mathrm{N}$ e $\mathrm{P}$ pela $P$. glomerata foram analisados pelo teste $\mathrm{F}$ e, para o caso de diferenças significativas, foi realizada a análise de regressão linear múltipla utilizando-se o aplicativo computacional Statistica 8.0. A superfície de resposta foi ajustada para os fatores $\mathrm{N}$ e $\mathrm{P}$ nos casos em que a interação foi significativa $(p<0,05)$.

\section{RESULTADO E DISCUSSÃO}

Verificou-se efeito significativo $(p<0,01)$ das interações entre as doses de $\mathrm{Ne} P$, havendo aumento da eficiência de absorção de $\mathrm{N}$ na proporção em que se elevaram os teores de $\mathrm{N}$ no solo (Figura 1 ). Ajustando-se a superfície de resposta aos dados obteve-se, com a dose de $444,5 \mathrm{mg} \mathrm{vaso}^{-1}$ de $\mathrm{N} \mathrm{e}$ $206,5 \mathrm{mg} \mathrm{vaso}^{-1}$ de $P$, a máxima eficiência de absorção, ou seja, 39,6 mg N g ${ }^{-1}$ de massa seca de raiz (Figura 1).

Possivelmente a maior eficiência da absorção de $\mathrm{N}$ se deva ao maior desenvolvimento do sistema radicular, a partir do fornecimento de N, pois, Epstein \& Bloom (2006) registraram, ao analisar o crescimento de raízes que, a presença de $\mathrm{N}$ na solução nutritiva fez com que as raízes fossem estimuladas ao crescimento, sendo este estímulo de $14 \%$ a $29 \%$ em comparação a ausência de N. De acordo com resultados obtidos por Skrebsky et al. (2008) a omissão de N limitou a absorção deste elemento e também o crescimento de $P$. glomerata.

A EFA de $P$ apresentou efeito significativo $(p$ $<0,01$ ) da interação entre as doses de N e P. O ponto máximo da EFA de $P$ foi estimado com a dose de $317,6 \mathrm{mg}$ vaso $^{-1}$ de $\mathrm{Ne} 375,0 \mathrm{mg}$ vaso- ${ }^{-1}$ de $\mathrm{P}$, sendo $3,1 \mathrm{mg} \mathrm{g}^{-1}$ a máxima eficiência de absorção de $P$ (Figura 2). Pode-se observar que acima dessas doses de $\mathrm{N}$ e $\mathrm{P}$, que proporcionaram à máxima EFA de $\mathrm{P}$, ocorre redução na EFA de $P$ não havendo incremento na eficiência de absorção de $\mathrm{P}$ (Figura 2).

Para a nutrição mineral de plantas medicinais o P tem função importante no aumento dos teores de princípios ativos. Martins et al. (1998) observaram que

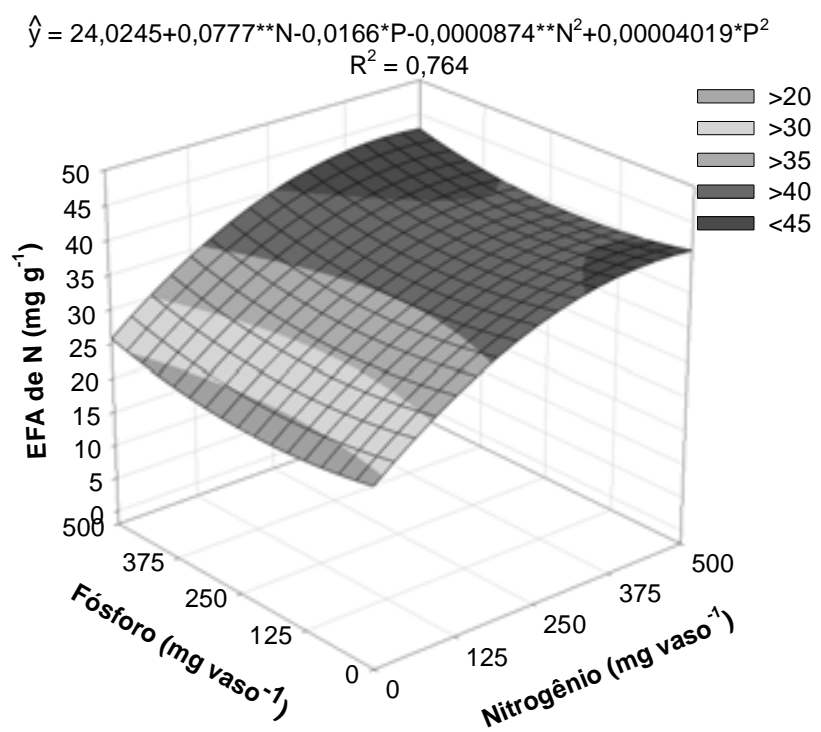

FIGURA 1. Superfície de resposta para a eficiência de absorção (EFA) de nitrogênio (N) em função dos níveis de $\mathrm{N}$, dentro de cada nível de fósforo $(\mathrm{P})$ aplicado. Dourados-MS, 2004. 
$\hat{y}=1,3879+0,0058^{* *} N+0,0043^{*} P-0,0000091316^{* *} N^{2}-0,0000057331^{*} P^{2}$

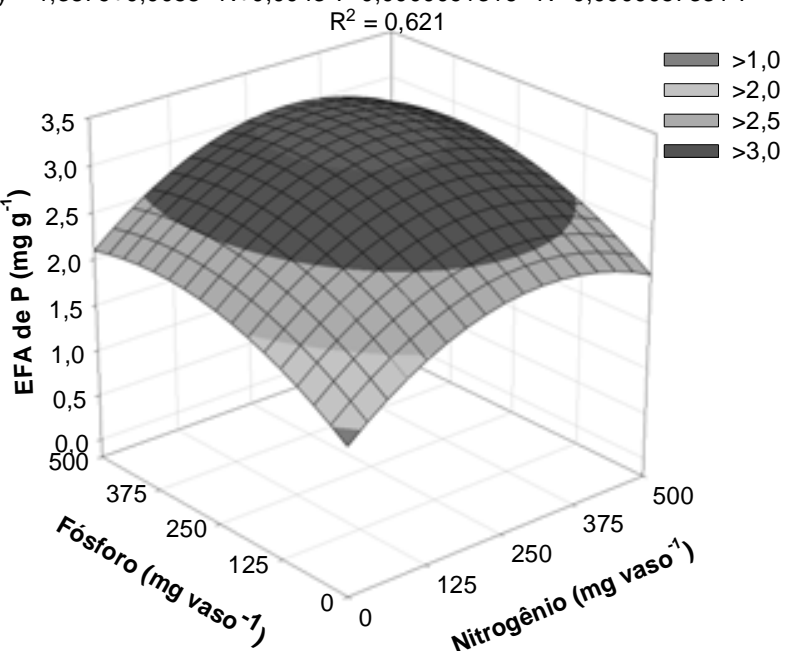

FIGURA 2. Superfície de resposta para a eficiência de absorção (EFA) de fósforo $(P)$ em função dos níveis de $\mathrm{N}$, dentro de cada nível de fósforo $(\mathrm{P})$ aplicado. Dourados-MS, 2004.

o fósforo pode contribuir para elevar a concentração de alcalóides em plantas medicinais e demais princípios ativos.

As plantas de Pfaffia glomerata apresentaram EFT máxima de $\mathrm{N}$ de $21,1 \%$, enquanto a EFT máxima de $P$ foi de $27,12 \%$ (Figuras 3 e 4 ) o que provavelmente é resultado da maior concentração de compostos nitrogenados - aminoácidos e proteínas - na parte radicular em detrimento da parte aérea. Essa espécie apresenta raízes tuberosas e geralmente bifurcadas com considerável produção de biomassa (Pott \& Pott, 1994). Houve significância da interação $N$ e $P(p<0,01)$ para a variável EFT de $P$. Com o aumento das doses de $P$ aplicadas no solo foi possível observar que a máxima EFT de $\mathrm{P}(27,1 \%)$ foi alcançada no ponto em que a dose de $\mathrm{P}$ foi de $441,6 \mathrm{mg} \mathrm{vaso}^{-1}$ e $247,1 \mathrm{mg} \mathrm{vaso}^{-1}$ de N. Dessa forma, com as maiores doses de $\mathrm{P}$ aplicadas ao solo, a eficiência de translocação de P elevou-se (Figura 4) e, mesmo em baixa concentração de $P$ no solo, $1 \mathrm{mg}$ $\mathrm{dm}^{-3}$, as plantas translocaram $\mathrm{P}$ da raiz para a parte aérea de forma significativa.

As plantas podem, em condições de baixo suprimento de $\mathrm{P}$, absorver o íon em maiores quantidades mantendo o crescimento do sistema radicular em detrimento da parte aérea. No caso do $P$ este fato já foi constatado por diferentes autores (Loneragan \& Asher, 1967; Martinez et al., 1993; Abichequer \& Bohnen, 1998; Alves et al., 1998). Uma das explicações para isto é que as raízes, estando mais próximas da fonte de suprimento, usam do limitado influxo destes nutrientes para as próprias necessidades de manutenção e crescimento (Fernandes, 2007).

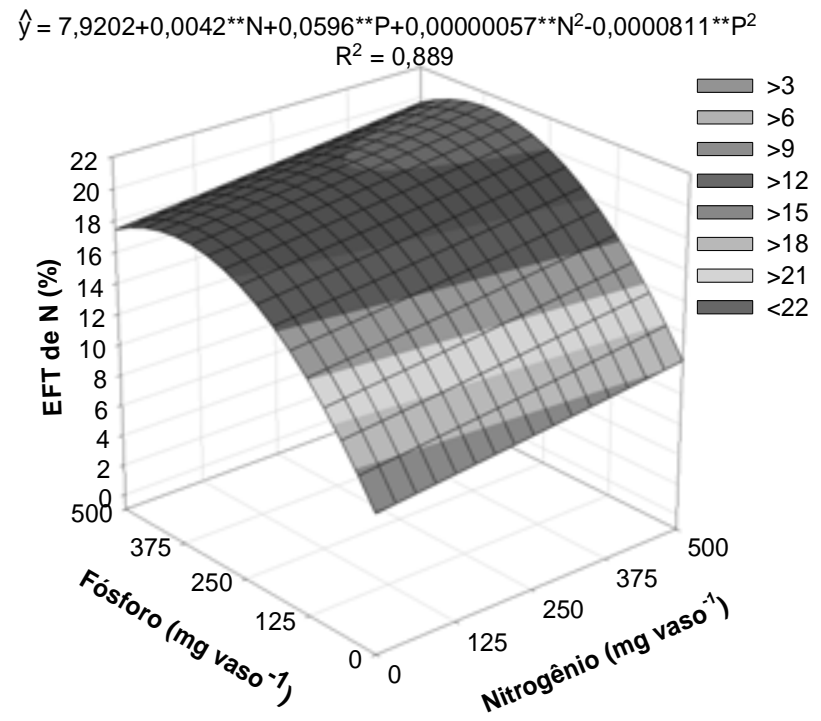

FIGURA 3. Superfície de resposta para a eficiência de translocação (EFT) de $\mathrm{N}$ em função dos níveis de nitrogênio $(\mathrm{N})$, dentro de cada nível de fósforo $(\mathrm{P})$ aplicado. Dourados-MS, 2004.

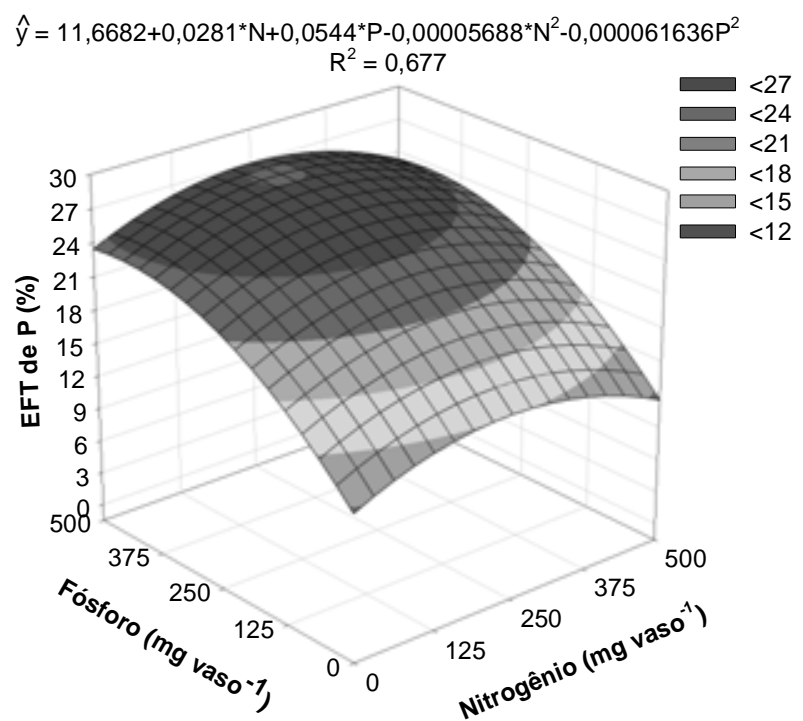

FIGURA 4. Superfície de resposta para a eficiência de translocação (EFT) de P em função dos níveis de nitrogênio $(N)$, dentro de cada nível de fósforo $(P)$ aplicado. Dourados-MS, 2004.

Aos dados da EFU de $\mathrm{Ne} \mathrm{P}$ foram ajustadas superfícies de resposta a partir da regressão da equação linear múltipla; dessa equação, calculou-se as doses dos fatores que representavam na superfície de resposta as estimativas mais adequadas de eficiência de uso de $\mathrm{N}\left(0,67 \mathrm{~g}^{2} \mathrm{mg}^{-1}\right)$, que nesse caso foi a estimativa do ponto de sela, projetadas para as doses de $\mathrm{N}$ e P de $364,8 \mathrm{mg} \mathrm{vaso}^{-1}$ e 324,0 $\mathrm{mg} \mathrm{vaso}^{-1}$, respectivamente (Figura 5).

Com relação à EFU de $\mathrm{P}$, observou-se efeito 
significativo $(p<0,05)$ da interação entre N e P (Figura 6). Essa maior eficiência de uso do $\mathrm{P}$ se deve à menor concentração desse elemento na planta (Figura 8) quando comparado com a concentração de N (Figura 6); com isso, a eficiência de uso do $P$ se torna maior.

Quanto menor for a concentração de um elemento na planta maior será a relação da produção de massa seca com o seu conteúdo; assim, comparando a eficiência de uso de N (Figura 5) com

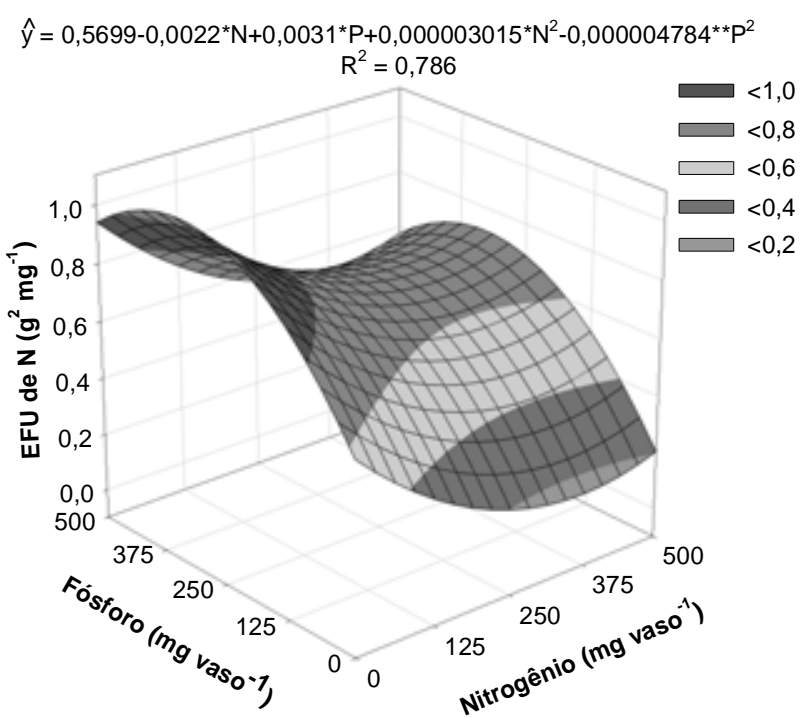

FIGURA 5. Superfície de resposta para a eficiência de uso (EFU) de nitrogênio (N) em função dos níveis de $\mathrm{N}$, dentro de cada nível de $\mathrm{P}$ aplicado. DouradosMS, 2004.

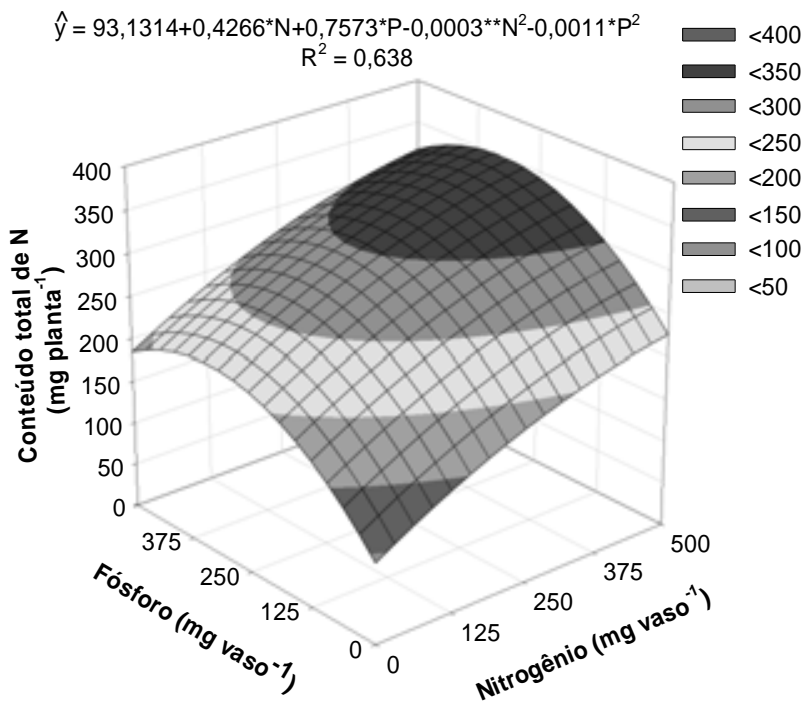

FIGURA 7. Superfície de resposta para o conteúdo total de nitrogênio $(\mathrm{N})$ em função dos níveis de $\mathrm{N}$, dentro de cada nível de $\mathrm{P}$ aplicado. Dourados-MS, 2004. a do $P$ (Figura 6), esse ultimo, por estar em menor concentração na planta, teve maior eficiência de uso.

Ao avaliar o conteúdo total de $\mathrm{N}$ pode se registrar que houve interação significativa $(p<0,05)$ entre os níveis de $\mathrm{N}$ e $\mathrm{P}$, sendo estimado o ponto máximo na superfície ( $361,8 \mathrm{mg}_{\text {planta-1 }}$ ) obtido com a dose de $500 \mathrm{mg}$ vaso-1 de $\mathrm{Ne} 344,2 \mathrm{mg}^{-1}$ vaso $^{-1}$ de $\mathrm{P}$ (Figura 7). Assim como o observado para o N, o conteúdo total de $\mathrm{P}$ na planta apresentou interação

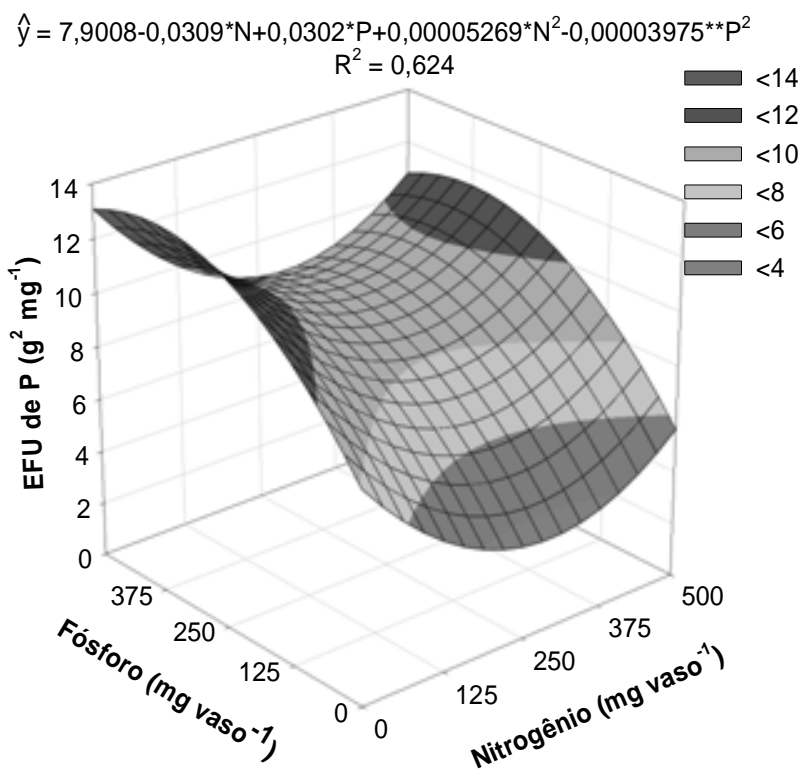

FIGURA 6. Superfície de resposta para a eficiência de uso (EFU) de fósforo (P) em função dos níveis de $\mathrm{N}$, dentro de cada nível de $\mathrm{P}$ aplicado. DouradosMS, 2004.

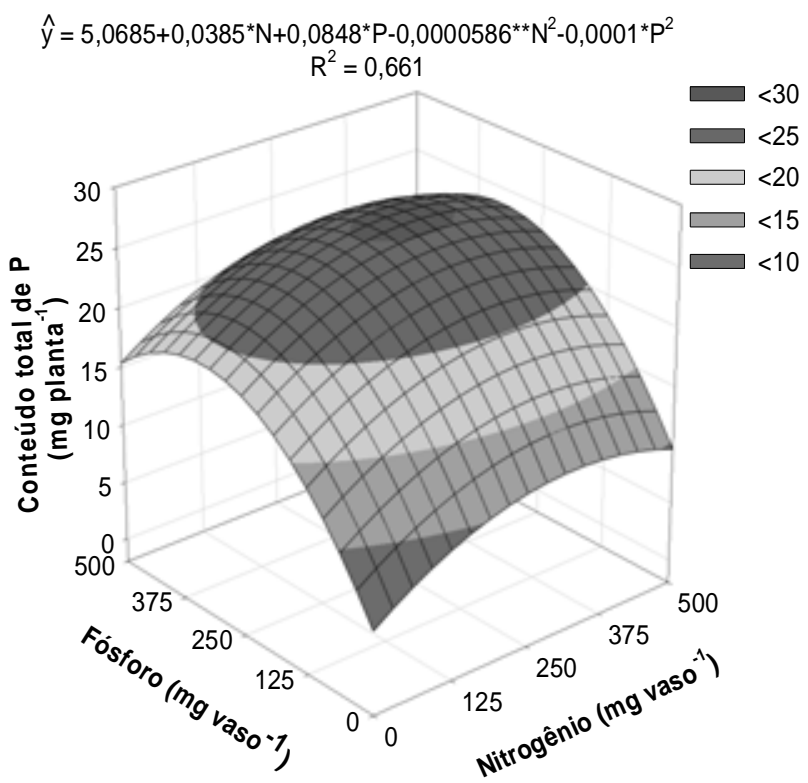

FIGURA 8. Superfície de resposta para o conteúdo total de fósforo $(P)$ em função dos níveis de $N$, dentro de cada nível de P aplicado. Dourados-ms, 2004. 
$(p<0,05)$ com ponto máximo na superfície de resposta igual a 29,4 mg planta-1 $^{-1}$ para a estimativa das doses de $\mathrm{N} \mathrm{e} P$ iguais a $328,5 \mathrm{mg}$ vaso-1 e $424,0 \mathrm{mg}^{-1}$ vaso $^{-1}$, respectivamente (Figura 8). Filho (2008) relata que com a adição de $\mathrm{N}$ ao solo há efeito interativo na absorção de $\mathrm{H}_{2} \mathrm{PO}_{4^{-}}$e que o teor de $\mathrm{P}$ na planta tem efeito positivo com aumento na concentração, o que leva a confirmar os resultados apresentados na Figura 8.

Pode-se concluir que a Pfaffia glomerata apresenta maior eficiência nutricional (EFA, EFT e EFU) e conteúdo total do $\mathrm{N}$ e $\mathrm{P}$, quando os mesmos são aplicados em conjunto no solo, havendo interação positiva entre esses nutrientes. A EFT de $\mathrm{N}$ é menor do que a do $P$, fato que se deve possivelmente a maior produção de compostos nitrogenados nas raízes da planta.

A eficiência nutricional máxima da $P$. glomerata foi obtida quando o $\mathrm{N}$ se encontrava na faixa de 247,1 a $500 \mathrm{mg} \mathrm{vaso}^{-1}$ e $\mathrm{P}$ na faixa de 206,5 a $441,6 \mathrm{mg} \mathrm{vaso}^{-1}$.

\section{REFERÊNCIA}

ABICHEQUER, A.D.; BOHNEN, H. Eficiência de absorção, translocação e utilização de fósforo por variedades de trigo. Revista Brasileira de Ciência do Solo, v.22, n.1, p.21-6, 1998.

ALVES, V.M.C. et al. Cinética e translocação de fósforo em híbridos de milho. Pesquisa Agropecuária Brasileira, v.33, n.7, p.1047-52, 1998.

CORRÊA JÚNIOR, C.C. et al. Sazonalidade na produção de raízes e teor de $\beta$-ecdisona em acessos de fáfia. Horticultura Brasileira, v.26, n.2, p.393-7, 2008.

EMBRAPA. Centro nacional de pesquisa de solos. Sistema brasileiro de classificação de solos. 5.ed. Rio de Janeiro: Editora EMBRAPA, 2006. 169p.

EMBRAPA. Centro Nacional de pesquisa de solos. Manual de métodos de análise de solo. 2.ed. Rio de Janeiro: Editora EMBRAPA, 1997. 212p.

EPSTEIN, E.; BLOOM, A. Nutrição mineral de plantas: princípios e perspectivas. 2.ed. Londrina: Editora Planta, 2006. 401p.

FENNER, R. et al. Hypnotic effect of ecdysterone isolated from Pfaffia glomerata (Spreng.) Pedersen. Brazilian Journal of Pharmacognosy, v.18, n.2, p.170-6, 2008. FERNANDES, A.R. et al. Produção de matéria seca e eficiência nutricional para $\mathrm{P}, \mathrm{Ca}$ e $\mathrm{Mg}$ em leguminosas herbáceas. Acta Amazonica, v.37, n.2, p.169-76, 2007. FLORES, R. et al. Análise de $\beta$-ecdisona em plantas in vivo e in vitro de Pfaffia glomerata (Spreng.) Pedersen, através da Cromatografia em Camada Delgada. Revista Brasileira de Plantas Medicinais, v.11, n.4, p.368-71, 2009a.
FLORES, R. et al. Extração de ecdisterona em raízes de ginseng brasileiro. Ciência Rural, v.39, n.4, p.1223-6, 2009b.

$\mathrm{LI}$, B. et al. Genetic variation in nitrogen use efficiency of loblolly pine seedlings. Science, v.37, n.2, p.613-26, 1991. LONERAGAN, J.F.; ASHER, C.J. Response of plants to phosphate concentration in solution culture: rate of phosphate absorption and its relation to growth. Soil Science, v.103, n.4, p.311-8, 1967.

MAGALHAES, P.M. Agrotecnología para el cultivo de fáfia o ginseng brasilero. In: MARTINEZ, J.V. et al. Fundamentos de agrotecnología de cultivo de plantas medicinales iberoamericanas. Santafé de Bogotá: Editora Convênio Andrés Bello/CYTED, 2000, p.323-32.

MALAVOLTA, E. Manual de nutrição de plantas. São Paulo: Editora Agronômica Ceres, 2006. 638p.

MALAVOLTA, E.; VITTI, G.C.; OLIVEIRA, S.A. Avaliação do estado nutricional das plantas: princípios e aplicações. Piracicaba: POTAFÓS, 1997. 319p.

MAIRAPETYAN, S.K. et al. Otimization of the N:P:K in the nutrient medium of some soilless aromatic and medicinal plants. Acta Horticutarae, v.502, n.3, p.29-32, 1999.

MARSCHNER, H. Mineral nutrition of higher plants. 2.ed. San Diego: Academic Press, 1995. 889p.

MARTINEZ, H.E.P. et al. Comportamento de variedades de soja cultivadas sob diferentes níveis de fósforo: Translocação do fósforo absorvido e eficiência nutricional. Revista Brasileira de Ciência do Solo, v.17, n.2, p.239-44, 1993.

MARTINS, E.R. et al. Plantas medicinais. Viçosa: UFV, imprensa universitária, 1998. p.220.

MONTANARI, I.J. et al. Influence of plantation density and cultivation cycle on root productivity and tenors of $\beta$ ecdysone in Pfaffia glomerata (Spreng.) Pedersen. Acta Horticulturae, v.3, n.1, p.125-9, 1999.

NOVAIS, R.F.; NUNES, F.N. Fósforo. In: NOVAIS, R.F. Fertilidade do solo. Viçosa: Sociedade Brasileira de Ciências do Solo, 2007. p.472-537.

POTT, A.; POTT. V.S. Plantas do Pantanal. Corumbá: EMBRAPA-SPI, 1994. 320p.

PRIMAVESI, A.C. et al. Absorção de cátions e ânions pelo capim-coastcross adubado com uréia e nitrato de amônio. Pesquisa Agropecuária Brasileira, v.40, n.3, p.247-53, 2005.

SKREBSKY, E.C. et al. Caracterização das exigências nutricionais de mudas de Pfaffia glomerata em Argissolo Vermelho distrófico arênico pela técnica do nutriente faltante. Ciência Rural, v.38, n.4, p.989-96, 2008.

SIDDIQI, M. et al. Utilization index: a modified approach to the estimation and comparison of nutrient utilization efficiency in plants. Journal Plant Nutrition, v.4, n.1, p.289302, 1981.

SIMÕES, C.M. et al. Farmacognosia: da planta ao medicamento. Editora UFRGS/ UFSC, 2000. 821p.

SWIADER, J.M. et al. Genotypic differences in nitrate uptake and utilization efficiency in pumpkin hybrids. Journal Plant Nutrition, v.17, n.3, p.1687-99, 1994.

Rev. Bras. PI. Med., Botucatu, v.14, n.2, p.255-260, 2012. 